\title{
Effects of Shang Ke Jie Gu tablet on fracture healing in rabbits
}

\author{
Yanhua Ma*, Fuyan Xie, Wa Gao \\ Traditional Chinese and Western Medicine Branch of the Third Affiliated Hospital of Inner Mongolia Medical University, \\ Baotou, China
}

Received: June 25, 2016

Accepted: July 25, 2016

Online Published: September 10, 2016

DOI: $10.14725 /$ dcc.v3n3p6

URL: http://dx.doi.org/10.14725/dcc.v3n3p6

\begin{abstract}
Objective: To study the effects of Shang Ke Jie Gu tablet on fracture healing in rabbits.

Methods: 40 New Zealand rabbits, half male and half female, were randomly divided into two groups: normal saline group (N group) and Shang Ke Jie Gu tablet group (S group). Each group consisted of 20 rabbits. Left radial fracture models in upper and middle sections were made in rabbits and then intra-gastric administration was given. Five rabbits in each group were killed on the $10^{t h}, 20^{t h}, 30^{t h}$ and $40^{t h}$ day after surgery, left radius was taken to get an $X$-ray. The degree of healing was assessed by the evaluation criteria of Shanghai Orthopaedics Institute.

Results: The degree of fracture healing in $\mathrm{S}$ group was higher in the comparison with that in $\mathrm{N}$ group.

Conclusions: Shang Ke Jie Gu tablet might affect endochondral ossification during fracture healing of rabbits, and promote porosis and fracture healing.
\end{abstract}

Key Words: Fracture healing, Shang Ke Jie Gu tablet, $X$-ray

Fracture is a very common clinical situation where the integrity and/or continuity of the bone is interrupted. Traditional Chinese medicine has a long history in the treatment of fracture. In this study, rabbits with deficiency in the radial bone were used as fracture models to observe the effect of Shang Ke Jie Gu tablet on fracture healing in rabbits.

\section{Materials and methods}

\subsection{Experimental animals}

40 New Zealand rabbits of 5-month old (born in May 2013) were chosen, weighed 1-2 kg, half male and half female, purchased from Animal Center of Inner Mongolia Medical University. The rabbits were fed with standard pelletized feed.

\subsection{Experimental methods}

\subsubsection{Animal grouping and model preparation}

40 rabbits were allowed to acclimate for 1 week and then modeled. The rabbits were randomly divided into two groups: S group (Shang Ke Jie Gu tablet group) and $\mathrm{N}$ group (normal saline group). Each group consisted of 20 rabbits. The rabbits received anesthesia through marginal ear vein by pentobarbital sodium solution with the mass fraction of $3 \%(1 \mathrm{mg} / \mathrm{kg})$. After anesthesia, rabbit hair on the front limbs was removal. Sterilized the surgical field and whisked sterile drapes. Anteromedial longitudinal incision was made from the upper, through the middle, to the lower section. The skin and hypodermis were incised layer by layer, and muscle tissues were separated by blunt dissection. $5 \mathrm{~mm}$ defect was made with 5 superposed saw blades around the termination where pronator teres was attached to the radius,

*Correspondence: Yanhua Ma; E-mail: dcc59@ncspress.com; Address: Traditional Chinese and Western Medicine Branch of the Third Affiliated Hospital of Inner Mongolia Medical University, Baotou, 014010, China. 
and left the ulna intact. Muscles and skin were sutured layer by layer and bound up with aseptic dressing without the bone fixed internally or externally. After surgery, the rabbits were given penicillin injections once a day $\left(4 \times 10^{4}\right.$ $\mathrm{U} / \mathrm{kg}$ ) by intramuscular for 3 days in order to prevent from infection.

\subsubsection{Methods of administration and dosage}

Drug suspension was given by intra-gastric administration. Rabbits in S group were given Shang Ke Jie Gu tablet with the dosage of $6 \mathrm{mg} / \mathrm{kg}$, which was dissolved by $10 \mathrm{ml}$ of normal saline. The rabbits in $\mathrm{N}$ group were given $10 \mathrm{ml}$ of normal saline in the whole course of the experiment.

\subsubsection{Specimen collection and detection indicators}

$X$-ray examinations were made respectively on the $10^{t h}$, $20^{t h}, 30^{t h}$ and $40^{t h}$ day after model establishment. All radiographs were taken with the standard radiographic technique from $45 \mathrm{kV}, 100 \mathrm{~mA}$, small focal length, $0.06 \mathrm{~s}$. The examination results were read by radiologists and the degree of fracture healing was assessed by the evaluation criteria of $X$-ray examination established by Shanghai Orthopaedics Institute.

\subsection{Statistical treatment}

SPSS17.0 was applied to statistical treatment, and rank sum test was used in the comparison of all categorical data between the two groups. The difference $(p<.05)$ was statistically significant.

\section{Results}

\subsection{Comparison of $X$-ray examination results in two groups of rabbits}

$X$-ray examinations were made respectively on the $10^{t h}$, $20^{t h}, 30^{t h}$ and $40^{t h}$ day. By comparative observation, the results showed that, callus density, the filling degree of bone defect and osteotylus quantity in $\mathrm{S}$ group were excelled than those in $\mathrm{N}$ group in the early and the middle stages of fracture healing, and in the late stage, callus remodeling was faster than that in $\mathrm{N}$ group (see Figure 1).
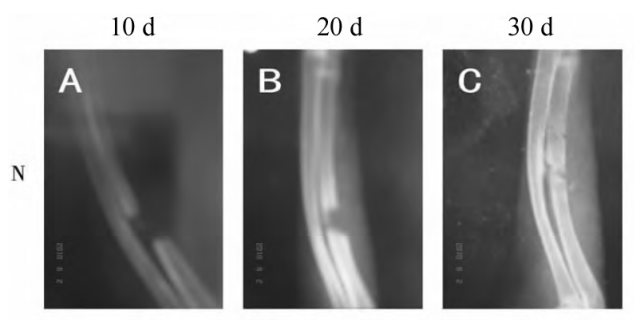

$40 \mathrm{~d}$
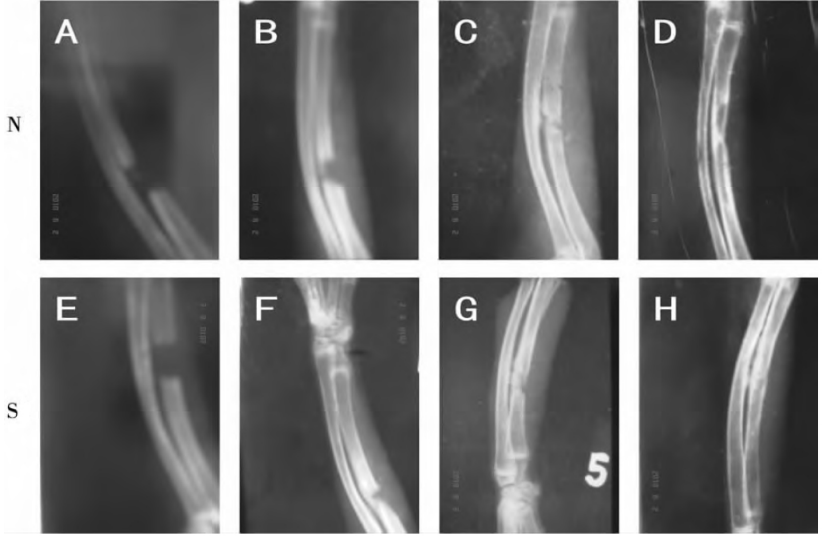

Figure 1: $X$-ray examination results of rabbits in two groups on different time points

\section{$2.2 X$-ray examination scoring}

Five rabbits in each group were required of $X$-ray examination on the $40^{t h}$ day. The degree of healing was assessed by the evaluation criteria of Shanghai Orthopaedics Institute. Rank sum test was used in the comparison between the two groups. Callus formed around the fracture end, periosteal reaction, callus structure, callus density and the margin of callus were included in the assessment of the degree of healing. Grades were represented by marks,,, \pm++++++ and ++++ . The degree of healing is better as the number of "+" mark increases. Results have showed that, callus density, the filling degree of bone defect and osteotylus quantity in $\mathrm{S}$ group were excelled than those in $\mathrm{N}$ group in the early and the middle stages of fracture healing, and in the late stage, callus remodeling was faster than that in $\mathrm{N}$ group (see Table 1).

Table 1: $X$-ray examination scoring (Grade) of left radiuses in rabbits of two groups on the $40^{t h}$ day after surgery (cases)

\begin{tabular}{lllllll}
\hline Group & Cases & $\mathbf{\pm}$ & + & ++ & +++ & ++++ \\
\hline N Group & 5 & 0 & 1 & 1 & 2 & 2 \\
S Group & 5 & 0 & 0 & 1 & $3^{*}$ & $3^{*}$ \\
\hline Note. In comparison with N group, ${ }^{*} p<.05$
\end{tabular}

\section{Discussion}

After fracture, the fracture end and its surrounding tissues will go through a series of stages such as inflammatory reaction, organized hematoma, cells proliferation and calcification, and then the continuity of the bone is recovered to the state of bone union. Fracture healing is a complicated problem, which is affected by various factors. ${ }^{[1]}$ Two ossification modes are involved in the process of fracture healing: intramembranous ossification and endochondral ossifi- 
cation. Intramembranous ossification starts with cells proliferation, i.e., osteoblasts inside and outside the periosteum begin to proliferate after bone fracture to make a connection with fracture ends. The periosteum grows in thickness and comes to be calcified. Inner and outer periosteum proliferation and calcification lead to the formation of internal calluses and external calluses respectively. The process of periosteal proliferation and calcification is defined as intramembranous ossification. Intramembranous ossification is an essential mechanism to the fracture healing of cortical bones. Endochondral ossification is defined as follows: fracture ends and blood clot resulted by hematoma in the medullary cavity, as well as injuries surrounding the fracture part and necrotic soft tissues mutually stimulate and produce aseptic inflammation and capillary proliferation. Capillaries, phagocytes, fibroblasts and other cells intrude into hematoma, which is gradually eliminated, organized and formed into granulation tissues. As granulation tissues come to mature, they are formed into fibrous tissues, and then transformed into temporary cartilage tissues. Osteoblasts intrude into the surrounding of cartilage tissues and replace cartilage cells. At this time, local cells secrete more phosphatases, under the action of which a large sum of phosphate radicals are released and combined with calcium ions to form calcium phosphate; original cartilage cells are transformed into bone tissues after deposition. As it is a process by which cartilage tissues are transformed into bone tissues, the process is referred as endochondral ossification. Endochondral ossification can lead to the formation of circular callus and intramedullary callus. Circular calluses are formed between fracture ends; and intramedullary calluses occupy the medullar cavity, which can be sealed by this type of calluses. However, the medullar cavity will be interconnected in the later remodeling process.

\section{References}

[1] Sun XJ. Factors affecting fracture healing. Guide of China Medicine. 2012; 10(17): 82-84.

[2] Qiao L, Wang J, Yuan ZM. Content determination of strychnine and
Shang Ke Jie $\mathrm{Gu}$ tablet contains various ingredients extracted from traditional Chinese medicine. Some studies have shown that it cannot only relieve tissue edema, bleeding and inflammatory cell infiltration, but also improve micro circulation around the fracture part. Meanwhile, it can facilitate blood supply around the injured part and contribute to the fracture healing. ${ }^{[2]}$ Other researches also have shown that, this drug can promote the deposition of calcium salt in bone matrix on the fracture part, and then facilitate the fracture healing. ${ }^{[3]}$ In this experiment, callus density, the filling degree of bone defect and osteotylus quantity in $\mathrm{S}$ group were excelled than those in $\mathrm{N}$ group in the early and the middle stages of fracture healing, and in the late stage, callus remodeling was faster than that in $\mathrm{N}$ group. On the $40^{\text {th }}$ day after surgery, the loss of fracture line could be observed in $\mathrm{S}$ group. Besides, external calluses were remodeled to compact bone cortex, ordered bone trabecula was found, and the medullar cavity was recovered to be interconnected basically. The mechanism is probably associated with the improvement of micro circulation and the acceleration of calcium salt deposition.

\section{Conclusions}

In conclusion, fracture healing is a complicated repair process of the organism, and it is affected by various factors. Shang Ke Jie Gu tablet can accelerate callus calcification in different stages of healing and promote the bone union.

\section{Conflicts of Interest Disclosure}

The authors have no conflicts of interest related to this article.

brucine in Shangkejiegu Tablet by HPLC. Guide of China Medicine. 2009; 6(31): 38-40.

[3] Liu HM, Xie FY. Effect of Shang Ke Jie Gu tablet on blood calcium, blood phosphatase and alkaline phosphatase in the process of fracture healing in rabbits. Chinese Community Doctors. 2013; 15(6): 28. 